\title{
Behavior of capacitive humidity sensors in monitoring the drying of concrete walls
}

\author{
Tuomas Raunima ${ }^{1, *}$, Eero Tuominen ${ }^{1}$, and Juha Vinha $^{1}$ \\ ${ }^{1}$ Tampere University, Building Physics, 33720 Tampere, Finland
}

\begin{abstract}
This research examines the behavior of capacitive humidity sensors in monitoring the drying of concrete walls in continuous measurements in laboratory conditions. Tests are carried out using continuous measuring of moisture with different capacitive sensors in concrete structures varied with three different types of thermal insulation materials. Sensors are sealed in plastic tubes that were preinstalled into the casting molds. Three borehole measurements are carried out as reference during the research. Results show differences in performance between the examined humidity sensors from two different manufacturers. The main difference is related to stability as sensors from the other manufacturer prove to be more prone to error. The study affirms that measuring humidity in concrete is challenging even when using high-quality humidity sensors.
\end{abstract}

\section{Introduction}

Evaluating the humidity of cast structures is a critical part of a construction stage. Drying times have a significant impact on building schedules and prolonged drying will delay the installation work. On the other hand, an adequate drying of structures before installation of insulating layers must be ensured. Premature insulation of structures often result in moisture damages and indoor air problems, which, in addition to reconstruction costs, may cause health problems to the users of the building. Therefore, a reliable measuring method is required to monitor the moisture of cast structures. [1]

Over time, several different methods have been used to evaluate the drying of cast structures. In borehole method, a humidity probe is left in a borehole until the humidity in the hole has reached an equilibrium state. The method is broadly used and reliable for measuring moisture in concrete structures and is the most common method in the Nordic countries. On the other hand, the method is affected by various uncertainty factors, which must be controlled in order to receive reliable measurements. Humid concrete is a demanding measurement environment for capacitive humidity sensors. [1-3]

A research on the drying of concrete walls was carried out previously at Tampere University [4]. The study monitored the moisture of cast concrete structures using capacitive humidity sensors with continuous measurements. During the study, inconsecutive phenomenons were discovered in the measured data, which led to doubts over the reliability of the measurements. The sensors were found to creep up to $10 \% \mathrm{RH}$

*Corresponding author: tuomas.raunima@tuni.fi 
when exposed to high humidity in continuous measurements. Due to problems found in previous study, this study focuses on the behavior of capacitive humidity sensors in similar experimental arrangements. The aim of the study is to investigate the behavior of capacitive humidity sensors in monitoring the drying of cast concrete walls by comparing side-by-side measuring devices of two different known manufacturers and using their different filter options.

\section{Test arrangements}

\subsection{Measuring devises}

The structure of the capacitive humidity sensor (Fig. 1) consists of two electrode plates and a hygroscopic polymer or metal oxide film between them. The medium film absorbs water from its environment and the amount of water absorbed is dependent on the humidity and temperature of the environment. The function of the sensor is based on measuring the change in capacitance between these electrode plates when the dielectric constant of the medium film changes with the change in humidity.

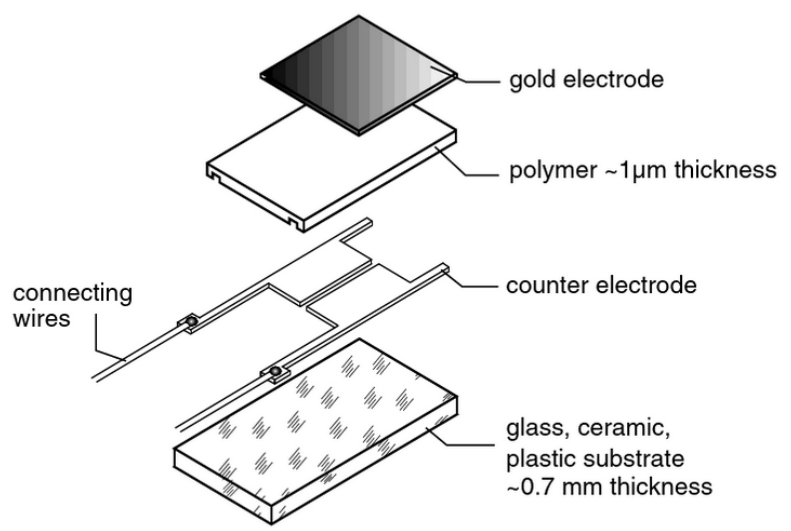

Fig. 1. Typical setup of a capacitive polymer sensor. [5]

This study compares the previously used Rotronic HC2-S probe with a polyethylene filter (PE) and a Teflon filter (PTFE), a Vaisala HMP110 probe with a Teflon filter (PTFE) and a sintered steel filter (S) along with Vaisala's older HMP230 probes with standard filters. At Tampere University, there was no previous experience from using the Rotronic probes for measuring moisture of concrete. HMP110 is Vaisala's "standard" probe and is widely used in the field in the Nordic countries for measuring humidity of concrete structures with borehole method obtaining consistent measuring data. The older HMP230 probes were included for the study because they were freed up from previous studies at Tampere University.

Rotronic HC2-S were purchased new and factory calibrated for previous studies, and they were carefully calibrated before adopting for this study. Vaisala HMP110 were purchased new and factory calibrated for this study. Vaisala HMP230 is an older probe that has been used in other studies over the years. They also were carefully calibrated before adopting for this study. The calibrations were performed using two calibration points for temperature $\left(5^{\circ} \mathrm{C}\right.$ and $\left.20{ }^{\circ} \mathrm{C}\right)$ and four calibration points for humidity using saline solutions in $20{ }^{\circ} \mathrm{C}$ temperature: $97 \% \mathrm{RH}\left(\mathrm{K}_{2} \mathrm{SO}_{4}\right), 85 \% \mathrm{RH}(\mathrm{KCl}), 57 \% \mathrm{RH}(\mathrm{NaBr})$ and $33 \% \mathrm{RH}$ 
$\left(\mathrm{MgCl}_{2}\right)$. During the research, control measurements were made based on at least two reference humidities and, if necessary, a probe that exceeded the margin of error was replaced with a calibrated unit.

\subsection{Test walls and measuring methods}

The layer thickness of the concrete structured shell in the wall elements was $150 \mathrm{~mm}$. Each test wall was thermal insulated on other side with either a $150 \mathrm{~mm}$ PIR, $200 \mathrm{~mm}$ EPS or $205 \mathrm{~mm}$ mineral wool insulation board (Fig. 2). The material and layer thickness of the insulation boards are the same as in the previous study to mimic the test arrangements. There were two test walls for each type of insulation. The humidity of the other was measured from cast-mounted measuring tubes and using borehole method based on measurements from one hole per measure. The humidity of the second test wall was measured by borehole measurements from two holes for the mineral wool insulated wall and three holes for the EPS- and PIR-insulated walls.
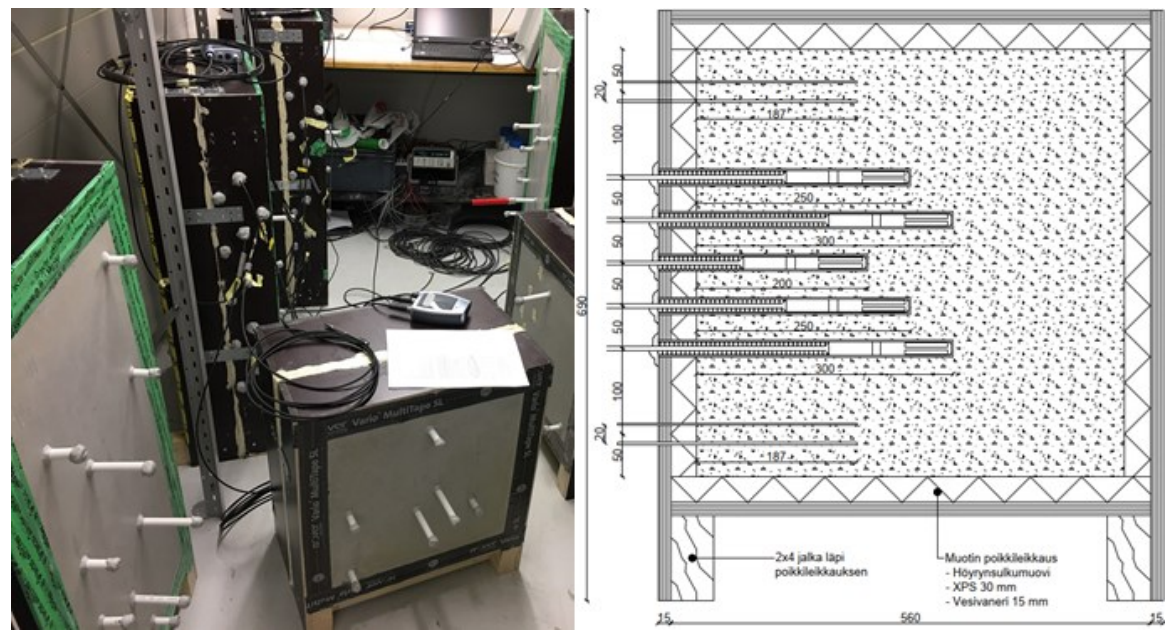

Fig. 2. Test walls in the laboratory and drawing of the mineral wool insulated test wall with measuring tubes.

Borehole measurements were carried out with Vaisala HMP110 probes and were used as reference to the cast-mounted measuring tubes in the study because the concerned measurement method and probes are widely used in the field. The chosen measuring depths of the $150 \mathrm{~mm}$ thick concrete shells are $25 \mathrm{~mm}, 75 \mathrm{~mm}$ and $125 \mathrm{~mm}$, corresponding to the measurement depths of the cast-mounted measuring tubes. The measurements were executed thrice during the research: 12, 41 and 191 days after casting. Reference graphs were drawn based on the measurements (Fig. 3). 


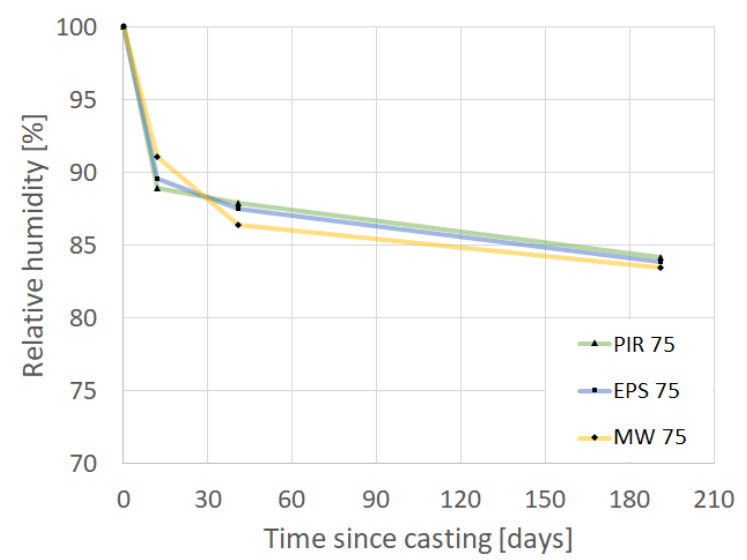

Fig. 3. Reference graphs based on the borehole measurements at measuring depth of $75 \mathrm{~mm}$.

From the measuring tubes, relative humidity was initially measured with short, maximum of 60 minutes stabilization time (Fig. 4). The goal was 60 minutes, but all measurements were aborted if any of the Rotronic probes reached a $100 \% \mathrm{RH}$ reading and the last recorded reading was used as measurement result. The reason for this was to protect the probes inside the concrete in humidity above $90 \% \mathrm{RH}$, which caused problems in the previous study [4]. To confirm the reliability of the sensors there were control measurements done 14, 38, 88, 129 and 181 days after casting.

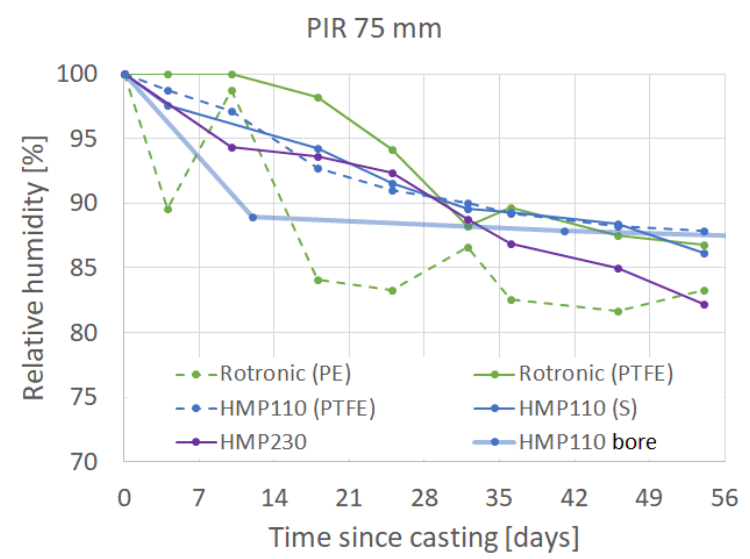

Fig. 4. Point-form measurements from PIR-insulated wall at measuring depth of $75 \mathrm{~mm}$.

Two consecutive continuous measuring periods were carried out after the measured relative humidity of the pore air in the concrete dropped below $90 \% \mathrm{RH}$ each lasting roughly a month (Fig. 5). The two measuring periods were timed 61-86 and 94-127 days after casting. The measured data was compared to the reference values obtained from the borehole measurements. During the second measuring period, malfunction in the test room caused the room temperature to rise and this affected the measured data. However, this gave more information on the stability and fault tolerance of the different sensors. 

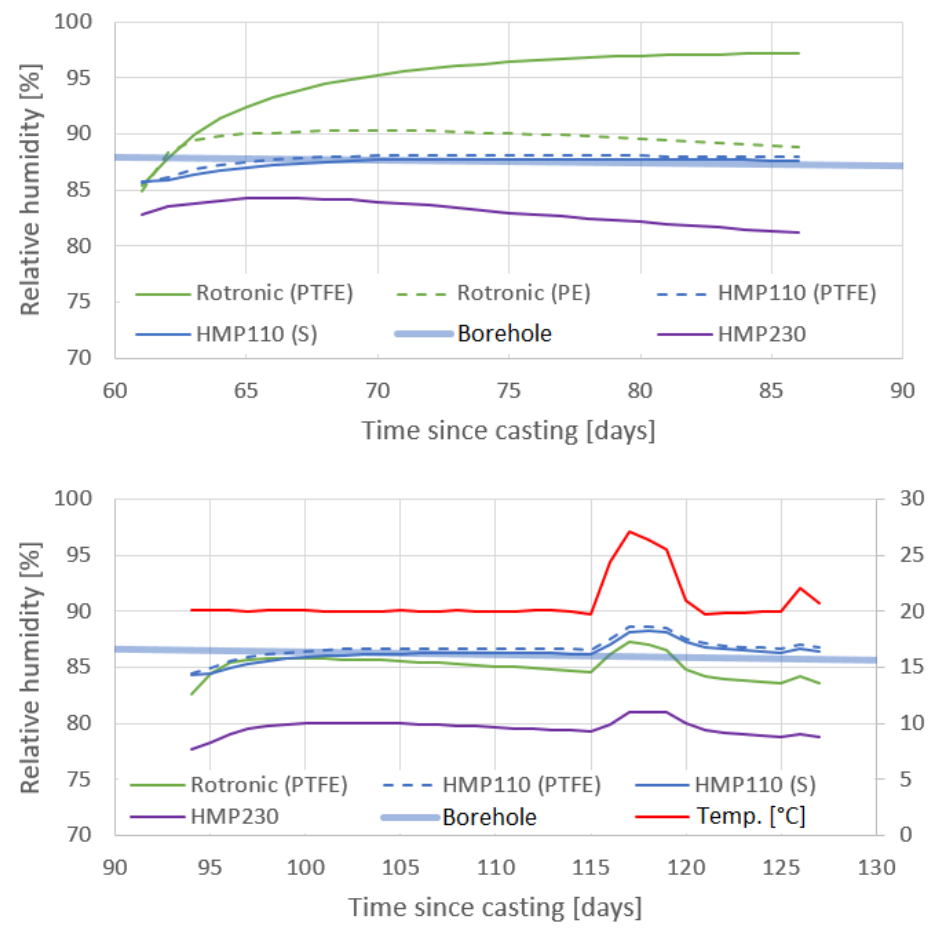

Fig. 5. Continuous measurements from PIR-insulated wall at measuring depth of $75 \mathrm{~mm}$.

\section{Results and conclusions}

Based on the study, reliable measurement of relative humidity of the pore air in concrete using continuous measurements in above $90 \%$ RH moist concrete with capacitive humidity sensors is difficult and the measurement results may contain significant flaws (Fig. 6). After the concrete had dried below $90 \% \mathrm{RH}$, the dispersion of the measured relative humidity results began to tighten close around the reference curve of the borehole measurements. The measurement deviation is caused by the used stabilization time in addition to the applied measuring method and the execution of the measurement. Based on the results, the use of cast-mounted measuring tubes increases error factors to the measurement.

Compared to borehole measurements, Vaisala HMP110 gave the most similar measurement results from the cast-mounted measuring tubes. The most significant deviation occurred in the initial phase of the measurements $(<30$ days $)$. The successive measurements of the Rotronic HC2-S probe contained more deviation and incorrect measurement results. However, based on prior experience, the Rotronic HC2-S is well suited for other applications. Inconsistent dispersion in measurement results is likely associated with highly moist alkaline concrete and condensation in the sensor. The problem is particularly pronounced when measurements are carried out under changing temperature conditions, for example at construction sites. 


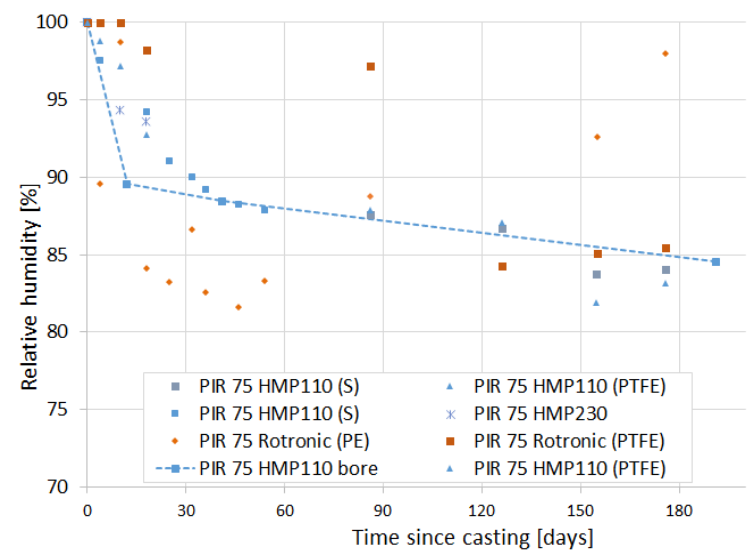

Fig. 6. Some measurement values from PIR-insulated wall at measuring depth of $75 \mathrm{~mm}$.

The benefit of using continuous measurements in relation to reliability with the studied concrete quality is questionable. In the initial stage, the measurement error and the risk of condensation are high. After the initial stage, the drying is so slow that the upward creeping of the measured values at its worst cancels out the simultaneous drying. In borehole method, the humidity evaporated from a drilled concrete surface is measured at the moment of examination. Therefore, the required stabilization time is shorter and thus the risk of measurement errors and condensation in the sensor is smaller. Based on the study, it is recommended to use point-form borehole measurements that require a short stabilization time to monitor the drying of concrete structures instead of continuous measurements from cast-mounted measuring tubes. If continuous measurements are to be used, it is recommended to mount the probes only when the temperature of the structure can be kept as constant as possible.

This work was supported by the European Regional Development Fund [grant number A70256]; Business Finland [grant number 4676/31/2014]; and 37 companies.

\section{References}

1. T. Merikallio, Evaluation of adequate drying of a concrete floor during construction process, Doctoral Thesis, Helsinki University of Technology, Helsinki, Finland (2009)

2. ASTM F2170-16b. Standard Test Method for Determining Relative Humidity in Concrete Floor Slabs Using in situ Probes, ASTM International, West Conshohocken, PA (2016) www.astm.org

3. RT 14-10984. Betonin suhteellisen kosteuden mittaus, Rakennustietosäätiö RTS (Building Information Foundation RTS sr), (2010) www.rakennustieto.fi

4. L. Korhonen, Drying examinations of prefabricated concrete walls insulated with cellular plastic, Master of Science Thesis, Tampere University, Building Physics, Tampere, Finland (2018)

5. R. Wernecke, J. Wernecke, Industrial Moisture and Humidity Measurement: A Practical Guide, John Wiley \& Sons, Incorporated (2013) 